\title{
Corneal Hysteresis as A Risk Factor for Optic Nerve Head Surface Depression and Retinal Nerve Fiber Layer Thinning in Glaucoma Patients
}

\section{Guihua Xu}

Huizhou Municipal Central Hospital

Zilin Chen ( $\sim$ boydongdong1@126.com )

Huizhou Municipal Central Hospital

\section{Research Article}

Keywords: $\mathrm{CH}, \mathrm{CSLO}, \mathrm{ONH}$, patients

Posted Date: January 19th, 2021

DOI: https://doi.org/10.21203/rs.3.rs-143919/v1

License: (c) (i) This work is licensed under a Creative Commons Attribution 4.0 International License.

Read Full License 


\section{Abstract}

Purpose: To evaluate the role of corneal hysteresis $(\mathrm{CH})$ as a risk factor for progressive $\mathrm{ONH}$ surface depression and RNFL thinning measured by confocal scanning laser ophthalmoscopy (CSLO) and spectral-domain optical coherence tomography (SD-OCT), respectively in glaucoma patients.

Design: Prospective study.

Methods: A total of 146 eyes of 90 patients with glaucoma were recruited consecutively. The $\mathrm{CH}$ measurements were acquired at baseline and 4-months interval using the Ocular Response Analyzer (Reichert Instruments, Depew, NY). Eyes were imaged by CSLO (Heidelberg Retinal Tomograph [HRT]; Heidelberg Engineering, GmbH, Dossenheim, Germany) and SD-OCT (Cirrus HD-OCT; Carl Zeiss Meditec $\mathrm{AG}$, Dublin, $\mathrm{CA}$ ) at approximately 4-month intervals for measurement of $\mathrm{ONH}$ surface topography and RNFL thickness, respectively. Significant ONH surface depression and RNFL thinning were defined with reference to Topographic Change Analysis (TCA) with HRT and Guided Progression Analysis (GPA) with Cirrus HD-OCT, respectively. Multivariate cox proportional hazards models were used to investigate whether $\mathrm{CH}$ is a risk factor for $\mathrm{ONH}$ surface depression and RNFL progression after adjusting potential confounding factors.

Results: All patients with glaucoma were followed for an average of 6.76 years (range, 4.56-7.61 years). Sixty-five glaucomatous eyes (44.5\%) of 49 patients showed ONH surface depression, 55 eyes (37.7\%) of 43 patients had progressive RNFL thinning and 20 eyes (13.7\%) of 17 patients had visual field progression. In the cox proportional hazards model, after adjusting baseline diastolic IOP, CCT, age, baseline disc area and baseline $\mathrm{MD}$, baseline $\mathrm{CH}$ was significantly associated with $\mathrm{ONH}$ surface depression and visual field progression ( $\mathrm{HR}=0.71, P=0.014$ and $\mathrm{HR}=0.54, P=0.018$, respectively), but not with RNFL thinning ( $\mathrm{HR}=1.03, P=0.836)$. For each 1-mmHg decrease in baseline $\mathrm{CH}$, the hazards for $\mathrm{ONH}$ surface depression increase by $29 \%$, and the hazards for visual field progression increase by $46 \%$.

Conclusions: The $\mathrm{CH}$ measurements were significantly associated with risk of glaucoma progression. Eyes with a lower $\mathrm{CH}$ were significantly associated with an increased risk of $\mathrm{ONH}$ surface depression and visual field progression in glaucoma patients.

\section{Introduction}

Glaucoma is chronic, progressive optic neuropathy with progressive nature. The structural change usually precedes the functional change during the course of glaucoma. Assessment of glaucoma progression, consideration with risk factor for structural change can help clinicians customize aggressiveness of therapy to increase the chances that patients will avoid functional impairment during their lifetime ${ }^{[1]}$. Identifiable optic never head (ONH) surface depression detected with HRT was shown to generally occur prior to identifiable RNFL thinning with OCT and visual field progression in previous study ${ }^{[2]}$. Nevertheless, a significant proportion of glaucomatous eyes showed ONH surface depression alone $(23.3 \%)$ or progressive RNFL thinning alone $(15.8 \%)^{[2]}$. It is unclear if the risk factors for development of 
ONH surface depression and progressive RNFL thinning are different. Investigation of risk factors for glaucoma progression has been largely based on visual field assessment and elevated IOP measurement has been shown in major clinical trials to be the most important risk factor for visual field progression in glaucoma patients ${ }^{[3-7]}$. In recent studies, corneal hysteresis $(\mathrm{CH})$ has also been related to visual field progression in glaucoma. $\mathrm{CH}$ is a measure of viscoelastic behavior of the cornea which can be measured clinically by ocular response analyzer (ORA, Reichert Instruments, Depew NY) ${ }^{[8]}$. $\mathrm{CH}$ reflects the ability to resist deformation applied to cornea. If the structural relationship between cornea and connective tissue in the posterior optic nerve head and lamina cribrosa exist, an association between $\mathrm{CH}$ and the functional behavior of the lamina cribrosa is conceivable, which may provide the basis that $\mathrm{CH}$ is related to glaucoma progression. Previous studies have shown that an eye with lower $\mathrm{CH}$ was associated with glaucoma progression ${ }^{[9-11]}$. In a retrospective study, Congdon et al ${ }^{[9]}$ showed that lower $\mathrm{CH}$ values, but not CCT, was associated with visual field progression. De Moraes et al reported an association between a lower $\mathrm{CH}$ and a faster visual field change ${ }^{[10]}$. In a recent study by Medeiros et al ${ }^{[11]}$, they showed that baseline $\mathrm{CH}$ was related to the rate of change of visual field index. It remains unknown, however, if $\mathrm{CH}$ is also a risk factor for progressive ONH surface depression and RNFL thinning. We hypothesized that the $\mathrm{CH}$ was related to $\mathrm{ONH}$ surface depression and RNLF thinning in addition to visual field progression. The purpose of this study was to investigate if $\mathrm{CH}$ measured at the baseline visit predicted progressive $\mathrm{ONH}$ surface depression and RNFL thinning in glaucoma patients.

\section{Results}

The study included 146 eyes of 90 patients with glaucoma followed for an average of $6.76 \pm 0.65$ years (range, 4.56-7.61 years). The baseline IOP was $18.8 \pm 3.3 \mathrm{mmHg}$ (range, 12.1-27.0 $\mathrm{mmHg}$ ) and baseline $\mathrm{CH}$ was $9.8 \pm 1.5 \mathrm{mmHg}$ (range, $6.7-14.2 \mathrm{mmHg}$ ). At the baseline examination, there were $68.7 \%$ mild $(M D \geq-6 \mathrm{~dB}), 22.5 \%$ moderate $(-6 \mathrm{~dB}>M D>-12 \mathrm{~dB})$ and $8.8 \%$ advanced $(M D \leq-12 \mathrm{~dB})$ glaucomatous eyes.

The demographics and baseline visual field, $\mathrm{ONH}, \mathrm{RNFL}$, and $\mathrm{CH}$ measurements of glaucoma patients are presented in Table 1. Figure 1 showing a scatter matrix illustration of the relationship among baseline IOP, CCT and $\mathrm{CH}$. A significant relationship was found between IOP and $\mathrm{CH}(\mathrm{r}=-0.19, P=0.018)$, and CCT and $\mathrm{CH}(\mathrm{r}=0.43, P<0.001)$. In this study, no significant relationship was found between age and $\mathrm{CH}(\mathrm{r}=0.016$, $P=0.129)$. 
Table 1

The demographics and baseline visual field, optic nerve head $(\mathrm{ONH})$, retinal nerve fiber layer (RNFL), and corneal hysteresis $(\mathrm{CH})$ measurements of glaucoma patients (Mean $\pm \mathrm{SD}$ )

\begin{tabular}{|ll|}
\hline Parameters & \\
\hline Patients/eyes & $90 / 146$ \\
\hline Age (years) & $54.05 \pm 15.11$ \\
\hline Gender, female (\%) & $35(38.9 \%)$ \\
\hline Refraction (D) & $-2.93 \pm 4.03$ \\
\hline Axial length (mm) & $24.78 \pm 2.07$ \\
\hline CCT $(\mu \mathrm{m})$ & $546.53 \pm 30.70$ \\
\hline Baseline diastolic IOP $(\mathrm{mmHg})$ & $18.76 \pm 3.34$ \\
\hline Baseline CH (mmHg) & $9.84 \pm 1.53$ \\
\hline Baseline RNFL $(\mu \mathrm{m})$ & $75.88 \pm 13.77$ \\
\hline Baseline MD $(\mathrm{dB})$ & $-4.90 \pm 5.00$ \\
\hline Baseline disc area $\left(\mathrm{mm}^{2}\right)$ & $2.22 \pm 0.53$ \\
\hline
\end{tabular}

SD - standard deviation; D - diopter; RNFL - retinal nerve fiber layer; IOP - intraocular pressure; CH corneal hysteresis; MD - mean deviation; CCT-central corneal thickness; $\mathrm{dB}$ - decibel

Sixty-five glaucomatous eyes (44.5\%) of 49 patients showed ONH surface depression, 55 eyes (37.7\%) of 43 patients had progressive RNFL thinning and 20 eyes (13.7\%) of 17 patients had visual field progression. Eyes with $\mathrm{ONH}$ surface depression had thinner CCT $(540.5 \pm 31.55 \mu \mathrm{m})$, younger age (45.05 \pm 15.06 years $)$ and smaller baseline $\mathrm{CH}(9.44 \pm 1.36 \mathrm{mmHg})$ than those without $\mathrm{ONH}$ surface depression (551.41 $\pm 29.29 \mu \mathrm{m}, 50.47 \pm 14.33$ years, $10.15 \pm 1.59 \mathrm{mmHg}$, respectively) (Table 2). No significant differences in axial length, baseline IOP, baseline RNFL thickness, MD and disc area $(P \geq 0.174)$ were found between eyes with and without ONH surface depression (Table 2). Eyes detected with RNFL progression had a higher baseline diastolic IOP than eyes without $(19.49 \pm 3.35 \mathrm{mmHg}$ verse $18.31 \pm$ $3.27 \mathrm{mmHg}, P=0.035$ ) (Table 3 ) whereas eyes detected with visual field progression had a smaller baseline $\mathrm{CH}(9.15 \pm 1.59 \mathrm{mmHg}$ and $9.95 \pm 1.49 \mathrm{mmHg}$, respectively, $P=0.027)$ and eyes with visual field progression had relative younger ages than those without visual field progression ( $47.90 \pm 17.32$ years and $48.10 \pm 14.51$ years, respectively, $P>0.05$ ), although the comparison was not significantly different (Table 4). 
Table 2

Comparisons of baseline ocular biometry, visual field, optic nerve head $(\mathrm{ONH})$, retinal nerve fiber layer (RNFL), corneal hysteresis (CH) and intraocular pressure (IOP) measurements between eyes with and without optic nerve head (ONH) surface depression with CSLO

\begin{tabular}{|llll|}
\hline & $\begin{array}{l}\text { With ONH surface } \\
\text { depression } \\
(\mathbf{6 5} \text { eyes) }\end{array}$ & $\begin{array}{l}\text { Without ONH surface } \\
\text { depression } \\
(\mathbf{8 1} \text { eyes) }\end{array}$ & $P^{*}$ \\
\hline Age (years) & $45.08 \pm 15.06$ & $50.47 \pm 14.33$ & 0.027 \\
\hline Axial length $(\mathrm{mm})$ & $25.08 \pm 1.75$ & $24.54 \pm 2.8$ & 0.174 \\
\hline CCT $(\mu \mathrm{m})$ & $540.45 \pm 31.55$ & $551.41 \pm 29.29$ & 0.029 \\
\hline $\begin{array}{l}\text { Baseline diastolic IOP } \\
\text { (mmH) }\end{array}$ & $18.38 \pm 3.26$ & $19.05 \pm 3.39$ & 0.224 \\
\hline Baseline CH (mmHg) & $9.44 \pm 1.36$ & $10.15 \pm 1.59$ & 0.028 \\
\hline $\begin{array}{l}\text { Baseline RNFL thickness } \\
(\mu \mathrm{m})\end{array}$ & $75.86 \pm 12.79$ & $77.31 \pm 14.24$ & 0.555 \\
\hline Baseline MD (dB) & $-4.74 \pm 5.24$ & $-5.03 \pm 4.82$ & 0.514 \\
\hline Baseline disc area $\left(\mathrm{mm}^{2}\right)$ & $2.04 \pm 0.42$ & $2.02 \pm 0.43$ & 0.929 \\
\hline
\end{tabular}

CCT-central corneal thickness; IOP - intraocular pressure; MD - mean deviation; $\mathrm{CH}$ - corneal hysteresis *Comparisons were performed using Linear mixed modeling 
Table 3

Comparisons of baseline ocular biometry, visual field, optic nerve head $(\mathrm{ONH})$, retinal nerve fiber layer (RNFL), corneal hysteresis (CH) and intraocular pressure (IOP) measurements between eyes with and without RNFL progression with Cirrus HD-OCT

\begin{tabular}{|llll|}
\hline & $\begin{array}{l}\text { With RNFL } \\
\text { progression } \\
(\mathbf{5 5} \text { eyes) }\end{array}$ & $\begin{array}{l}\text { Without RNFL progression } \\
\text { (91 eyes) }\end{array}$ & $P^{*}$ \\
\hline Age (years) & $49.33 \pm 16.49$ & $47.31 \pm 13.82$ & 1.000 \\
\hline Axial length (mm) & $24.71 \pm 2.02$ & $24.82 \pm 2.12$ & 0.860 \\
\hline CCT $(\mu \mathrm{m})$ & $550.71 \pm 31.22$ & $544.00 \pm 30.28$ & 0.197 \\
\hline Baseline diastolic IOP $(\mathrm{mmHg})$ & $19.49 \pm 3.35$ & $18.31 \pm 3.27$ & 0.035 \\
\hline Baseline CH (mmHg) & $9.92 \pm 1.48$ & $9.79 \pm 1.56$ & 0.587 \\
\hline Baseline RNFL thickness $(\mu \mathrm{m})$ & $78.40 \pm 12.74$ & $75.62 \pm 14.04$ & 0.366 \\
\hline Baseline MD (dB) & $-4.60 \pm 4.18$ & $-5.08 \pm 5.45$ & 0.514 \\
\hline Baseline disc area $\left(\mathrm{mm}^{2}\right)$ & $2.03 \pm 0.43$ & $2.03 \pm 0.42$ & 0.794 \\
\hline
\end{tabular}

CCT-central corneal thickness; IOP - intraocular pressure; MD - mean deviation; $\mathrm{CH}$ - corneal hysteresis *Comparisons were performed using Linear mixed modeling

Table 4 Comparisons of baseline ocular biometry, visual field, optic nerve head (ONH), retinal nerve fiber layer (RNFL), corneal hysteresis ( $\mathrm{CH}$ ) and intraocular pressure (IOP) measurements between eyes with and without visual field progression 


\begin{tabular}{|llll|}
\hline & $\begin{array}{l}\text { With visual field } \\
\text { progression } \\
(20 \text { eyes })\end{array}$ & $\begin{array}{l}\text { Without visual field } \\
\text { progression } \\
(126 \text { eyes })\end{array}$ & $P^{\star}$ \\
\hline Age (years) & $47.90 \pm 17.32$ & $48.10 \pm 14.51$ & 1.000 \\
\hline Axial length $(\mathrm{mm})$ & $25.03 \pm 2.04$ & $24.74 \pm 2.09$ & 0.059 \\
\hline CCT $(\mu \mathrm{m})$ & $551.15 \pm 32.39$ & $545.79 \pm 30.50$ & 0.466 \\
\hline $\begin{array}{l}\text { Baseline diastolic IOP } \\
(\mathrm{mmHg})\end{array}$ & $19.63 \pm 3.70$ & $18.62 \pm 3.27$ & 0.616 \\
\hline $\begin{array}{l}\text { Baseline CH (mmHg) } \\
\text { Baseline RNFL thickness }\end{array}$ & $9.15 \pm 1.59$ & $9.95 \pm 1.49$ & 0.027 \\
\hline$\mu$ m) & $77.15 \pm 10.37$ & $76.59 \pm 14.06$ & 0.617 \\
\hline Baseline MD (dB) & $-5.32 \pm 3.57$ & $-4.83 \pm 5.20$ & 0.652 \\
\hline Baseline disc area $\left(\mathrm{mm}^{2}\right)$ & $2.02 \pm 0.44$ & $2.03 \pm 0.43$ & 0.953 \\
\hline
\end{tabular}

CCT-central corneal thickness; IOP - intraocular pressure; MD - mean deviation; $\mathrm{CH}$ - corneal hysteresis

*Comparisons were performed using Linear mixed modeling

In the cox proportional hazards model, after adjusting baseline diastolic IOP, CCT, age, baseline disc area and baseline $\mathrm{MD}$, baseline $\mathrm{CH}$ was significantly associated with $\mathrm{ONH}$ surface depression and visual field progression ( $\mathrm{HR}=0.71, P=0.014$ and $\mathrm{HR}=0.54, P=0.018$, respectively) (Table 5). For each 1-mmHg decrease in baseline $\mathrm{CH}$, the hazards for $\mathrm{ONH}$ surface depression increase by $29 \%$, and the hazards for visual field progression increase by $46 \%$. 
Table 5

Cox proportional hazards models showing multivariate hazard ratios for optic nerve head $(\mathrm{ONH})$ surface depression, retinal nerve fiber layer (RNFL) progression and visual field progression after adjusting ages,

$\mathrm{CCT}$, baseline diastolic IOP, average IOP during follow-up, baseline disc area and baseline MD

\begin{tabular}{|c|c|c|c|c|c|c|}
\hline & \multicolumn{2}{|c|}{$\begin{array}{l}\text { ONH surface } \\
\text { depression }\end{array}$} & \multicolumn{2}{|c|}{$\begin{array}{l}\text { RNFL } \\
\text { progression }\end{array}$} & \multicolumn{2}{|c|}{$\begin{array}{l}\text { Visual field } \\
\text { progression }\end{array}$} \\
\hline & $\begin{array}{l}\text { Hazard } \\
\text { Ratio }\end{array}$ & $p$ & $\begin{array}{l}\text { Hazard } \\
\text { Ratio }\end{array}$ & $p$ & $\begin{array}{l}\text { Hazard } \\
\text { Ratio }\end{array}$ & $p$ \\
\hline Age (years) & 0.98 & 0.067 & 1.01 & 0.364 & 1.00 & 0.993 \\
\hline ССТ $(\mu \mathrm{m})$ & 0.99 & 0.270 & 1.01 & 0.407 & 1.02 & 0.058 \\
\hline $\begin{array}{l}\text { Baseline diastolic IOP } \\
(\mathrm{mmHg})\end{array}$ & 0.88 & 0.104 & 1.07 & 0.331 & 1.00 & 0.967 \\
\hline $\begin{array}{l}\text { Average of IOP during } \\
\text { follow-up (mmHg) }\end{array}$ & 1.10 & 0.275 & 1.06 & 0.474 & 1.03 & 0.808 \\
\hline Baseline $\mathrm{CH}(\mathrm{mmHg})$ & 0.71 & 0.014 & 1.03 & 0.836 & 0.54 & 0.018 \\
\hline Baseline disc area $\left(\mathrm{mm}^{2}\right)$ & 1.56 & 0.265 & 1.12 & 0.770 & 0.68 & 0.605 \\
\hline Baseline MD (dB) & 1.05 & 0.175 & 1.01 & 0.851 & 1.02 & 0.719 \\
\hline
\end{tabular}

\section{Discussion}

In this prospective study, we demonstrated that $\mathrm{CH}$ was significantly associated with $\mathrm{ONH}$ surface depression and visual field progression, but not with RNFL thinning in glaucoma patients. Eyes with a lower baseline $\mathrm{CH}$ were associated with a significantly higher risk of progressive $\mathrm{ONH}$ surface depression detected with CSLO and visual field progression detected with standard automated perimetry. Our finding showed that the ocular biomechanical properties are related to glaucoma progression and that measurement of corneal hysteresis is useful for risk assessment in glaucoma patients.

A number of studies have reported the association between $\mathrm{CH}$ and glaucomatous optic nerve head damage ${ }^{[12-14]}$. Prata et al showed that a lower $\mathrm{CH}$ value was associated with larger and deeper cup in untreated glaucoma patients ${ }^{[14]}$ and correlated with greater pressure-related rim and cup volume/ area change ${ }^{[15]}$. It has been suggested that optic nerve head and peripapillary sclera is more susceptible to IOP damage in eyes with a lower $\mathrm{CH}^{[12,14,16]}$. The association between baseline $\mathrm{CH}$ and visual field progression in glaucoma patients has also been shown by Medeiros et al. To our knowledge, this is the first study reporting the significant association between $\mathrm{CH}$ and $\mathrm{ONH}$ surface depression and RNFL progression in glaucoma. 
We showed that eyes with $\mathrm{ONH}$ surface depression (65 eyes) had smaller baseline $\mathrm{CH}(9.44 \pm$ $1.36 \mathrm{mmHg}$ ) than those without $\mathrm{ONH}$ surface depression $(10.15 \pm 1.59 \mathrm{mmHg})$. This finding appears to be counter-intuitive because a high $\mathrm{CH}$ is likely to be associated with a higher damping capacity to any force applied to the cornea. Assuming that there is an association in the ocular biomechanical properties between the cornea and the $\mathrm{ONH}$ and/or lamina cribrosa (LC) surfaces, we expected eyes with a lower $\mathrm{CH}$ would be associated with decreasing capacity to force applied to the optic nerve head. Optic nerve head surface will not tend to depress under a low $\mathrm{CH}$ situation, which would be associated with a lower risk of progressive $\mathrm{ONH}$ surface depression. However, it is notable that we were not able to differentiate plastic (permanent) from elastic (reversible) ONH surface deformation in this study. Although eyes with a high $\mathrm{CH}$ might be associated with a more deformable $\mathrm{ONH}$ and/or lamina cribrosa surfaces, these changes could be elastic. We speculated that eyes with a lower $\mathrm{CH}$ could be more susceptible to plastic deformation of $\mathrm{ONH}$ surface. Differentiating elastic from plastic $\mathrm{ONH}$ surface deformation would be relevant to further investigate the impact of $\mathrm{CH}$ measurement on glaucoma progression.

It is worth noting that $\mathrm{CH}$ measurement can be affected by other biometric variables, like CCT, age and IOP $[17,18]$. In our study, baseline $\mathrm{CH}$ was significantly associated with baseline IOP and CCT $(P \leq 0.018)$. We therefore included these parameters in the cox proportional hazards model. For each mmHg decrease in baseline $\mathrm{CH}$, the hazards for $\mathrm{ONH}$ surface depression increased by $29 \%$ after adjustment of baseline diastolic IOP, CCT, age, baseline disc area and baseline MD. However, baseline $\mathrm{CH}$ was not associated with progressive RNFL thinning. Of note, the LC and peripapillary sclera are the main load-bearing structures of the $\mathrm{ONH}^{[19]}$. Progressive changes of the peripapillary RNFL may be less directly related to the ocular biomechanical properties.

Medeiros et al ${ }^{[11]}$ performed a prospective study evaluating the role of $\mathrm{CH}$ as a risk factor for visual field progression in 114 eyes of 68 patients with glaucoma. They showed that a smaller baseline $\mathrm{CH}$ was significantly associated with a faster rate of visual field loss. In our study, visual field progression was determined based on the EMGT criteria and we obtained similar results. For each mmHg decrease in $\mathrm{CH}$, the hazards for visual field progression increased by $46 \%$. Our results corroborate the previous studies supporting that $\mathrm{CH}$ is a risk factor for visual field progression in glaucoma.

There are limitations of the study. First, the ability to detect progressive RNFL thinning, ONH surface depression and visual field progression could be related to the stage of glaucoma and the precision of the instrument. Second, there was no reference standard to validate and determine the sensitivity of RNFL and $\mathrm{ONH}$ changes detected by OCT and CSLO. Third, $\mathrm{CH}$ only represents a surrogate measure of the material properties of the cornea. It is not a direct measure of "corneal hysteresis". It is also unknown to what degree the material property of the cornea is related to that of the ONH structures. Finally, almost all patients in our study had received IOP lowering treatment. The sequence of RNFL, ONH and visual field changes in the natural course of glaucoma progression remains obscure.

In conclusion, eyes with a lower $\mathrm{CH}$ were significantly associated with an increased risk of $\mathrm{ONH}$ surface depression and visual field progression in glaucoma patients. This prospective study provides data 
supporting that baseline $\mathrm{CH}$ measurement is useful for evaluation of disease progression in glaucoma patients.

\section{Materials And Methods}

One hundred and forty-six eyes of 90 glaucoma patients were recruited with follow-up duration (mean: $6.76 \pm 0.65$ years; range: $4.56-7.61$ years). All patients were followed at approximately 4-month intervals during the period from June 2007 to December 2014 at the University Eye Centre, the Chinese University of Hong Kong. The detailed protocols in accordance with the Declaration of Helsinki were approved by the institutional review board of The Chinese University of HongKong. All subjects were told of the purpose of the study and gave written informed consent before inclusion. At each follow-up visit, subjects underwent a full ophthalmic examination, visual field examination, RNFL imaging with OCT, and optic nerve head with CSLO. We confirm that all methods were carried out in accordance with clinical research guidelines and regulations of The Chinese University of HongKong. All included eyes had best corrected visual acuity of at least 20/40. Eyes with retinal pathology, macular disease and refractive or retinal surgery were excluded. Eyes with uncomplicated cataract or glaucoma filtration surgery could be included in this study. Patients with glaucoma were identified on the basis of the presence of visual field defects with corresponding optic disc and RNFL changes in at least 1 eye independent of the level of IOP. During the follow-up, patients were treated at the discretion of the attending ophthalmologists with reference to the target IOP. Progressive RNFL thinning and ONH surface depression was detected with GPA (Carl Zeiss Meditec) and TCA (Heidelberg Engineering), respectively.

\section{Corneal Hysteresis Measurement with Ocular Response Analyzer}

Corneal hysteresis was acquired with ocular response analyzer (ORA, Reichert Inc, Depew, NY). For ORA measurement, a metered air pulse is delivered to the cornea and the cornea is flattened at two different time points (force-in applanation and force-out applanation). An electro-optical collimation detector system is used to record the light reflectivity from the cornea. The reflectivity is most intense when the cornea is completely flattened. $\mathrm{CH}$ is derived from the difference between the force-in and force-out applanation ${ }^{[20]}$. Three other parameters were also measured from each ORA signal profile: IOPcc, IOPg and CRF. Four ORA measurements were taken on an examined eye each time and the average value was calculated; only measurements with a waveform score larger than 5 were accepted.

\section{IOP Measurement with Dynamic Contour Tonometry}

IOP measurement was taken by dynamic contour tonometry (DCT) (Pascal; Swiss Microtechnology AG, Port, Switzerland). The working principle of DCT has been described ${ }^{[21]}$. In brief, the matching of the contour of the tonometer and the contour of the cornea allows the IOP measured by a pressure sensor located at the tip of the tonometer. In this study, the recording duration of each measurement was about 5 seconds. Measurement with a quality score less than 3 (scale 1 to 5 ) was repeated until 2 measurements of quality score between 1 and 3 (as recommended by the manufacturer) were obtained in each eye and 
the average value was calculated. DCT measures the systolic IOP and diastolic IOP and only diastolic IOP was analyzed in the study.

\section{Optical Coherence Tomography Imaging}

Spectral domain OCT imaging was performed with Cirrus HD-OCT (software version 5.0; Carl Zeiss Meditec). The acquisition rate of the Cirrus HD-OCT was 27,000 A-scans per second and the transverse and axial resolutions were $15 \mu \mathrm{m}$ and $5 \mu \mathrm{m}$, respectively. An optic disc cube consisting $200 \times 200$ axial scans, $6 \times 6 \mathrm{~mm}^{2}$ centered on the optic disc was used to evaluate the optic disc parameters for every subject. Images were captured by operators with at least 1 years' experience using the Cirrus HD-OCT (Carl Zeiss Meditec). The pupils were not routinely dilated during RNFL imaging. However, dilation with tropicamide $0.5 \%$ and phenylephrine $0.5 \%$ each was performed when the pupil size was too small for images of the required quality to be obtained. Images with poor centration, motion artifact, poor focus, or missing data were detected by the operator at the time of imaging, with rescanning performed in the same visit. Each OCT scan included in the study had signal strength $\geq 7$. Saccadic eye movement was detected with the line-scanning ophthalmoscope overlaid with OCT en face during OCT imaging. Images with motion artifact were rescanned in the same visit ${ }^{[22,23]}$. Serial RNFL thickness maps were analyzed for detection of change using the Guided Progression Analysis (GPA) ${ }^{[24]}$.

The Cirrus HD-OCT Guided Progression Analysis (GPA) (Carl Zeiss Meditec AG) was used to analyze serial RNFL thickness maps $(200 \times 200$ pixels) for detection of progressive RNFL thinning. Guided Progression Analysis automatically aligned and registered 2 baseline and the follow-up OCT images so that the same superpixel ( 1 superpixel $=4 \times 4$ pixels) locations could be analyzed for detection of change. The difference in RNFL measurement of an individual superpixel between the baseline and the follow-up RNFL thickness maps was compared with an estimate of test-retest variability of that particular superpixel (proprietary database from Carl Zeiss Meditec AG). Superpixels with an RNFL measurement difference exceeding the test-retest variability between a follow-up and the first and second baseline images would be encoded in yellow in the OCT RNFL thickness change map ( $50 \times 50$ superpixels). If the same changes were evident in an additional consecutive follow-up image, the superpixels would be encoded in red. In this study, the 2 baseline images were separated by approximately 4 months and progressive RNFL thinning was confirmed when an area of more than 20 superpixels (factory default) was encoded in red in the RNFL thickness change map for at least 2 consecutive visits. At least 3 consecutive follow-up visits showing significant RNFL thickness reduction were required to confirm progressive RNFL thinning.

\section{Confocal Scanning Laser Ophthalmoscopy Imaging}

Optic disc imaging was performed with the HRT 3 (software version 3.0, Heidelberg Engineering). A three dimensional topographic image consisting of up to $384 \times 384 \times 64$ pixels was constructed from multiple focal planes axially along the optic nerve head. An average of three consecutive scans was obtained and aligned to compose a single mean topography for analysis. The optic disc margin was outlined by an 
experienced examiner on the mean topographic image. Once the contour line was drawn, the software automatically calculated all the optic disc measurements. The reference plane is defined at $50 \mu \mathrm{m}$ posterior to the mean retinal height between $350^{\circ}$ and $356^{\circ}$ along the contour line. The area above and below the reference plane is defined as rim and cup respectively. Re-scanning was performed in the same visit if motion artifacts were detected immediately after the imaging. All eyes included in the analysis had an image quality standard deviation $\leq 30 \mu \mathrm{m}$.

The HRT Topographic Change Analysis (TCA, Heidelberg Engineering) was used to analyze serial ONH topography images $(96 \times 96$ superpixels; 1 superpixel $=4 \times 4$ pixels) for detection of $\mathrm{ONH}$ surface depression ${ }^{[25]}$. Individual superpixel $\mathrm{ONH}$ surface height measurements were compared between the baseline and each of the follow-up examinations with an $\mathrm{F}$ test. The pooled variability of the baseline and the follow-up examinations of a particular pixel was compared with the within variability of the baseline and the follow-up examinations (with an error probability of the F-test $<5 \%$ ). If significant $\mathrm{ONH}$ surface depression was detected in a superpixel and confirmed with at least 2 consecutive follow-up visits, the superpixel would be encoded in red in the significance map. The saturation of the color increased with the magnitude of surface height change. Progressive $\mathrm{ONH}$ surface depression was defined using 3 criteria (liberal, moderate, and conservative) with reference to the area and depth of ONH surface depression adopted from the studies by Chauhan and colleagues ${ }^{[26,27]}$. The liberal criterion required a cluster of $\geq 0.5 \%$ of the disc area and a depth change of $\geq 20 \mathrm{~mm}$; the moderate criterion a cluster of $\geq 1 \%$ of the disc area and a depth change of $\geq 50 \mathrm{~mm}$; and the conservative criterion a cluster of $\geq 2 \%$ of the disc area and a depth change of $\geq 100 \mathrm{~mm}$. In this study, the moderate criterion was adopted to ensure a fair specificity for ONH surface depression detection with HRT and RNFL thinning acquired with OCT [2]. The $\mathrm{ONH}$ surface depression was defined when the change was detected at least 3 of 4 consecutive follow-up examinations.

\section{Visual Field Examination}

Visual field was obtained with the white-on-white SITA standard 24-2 strategy in Humphrey Field Analyzer II-i (Carl Zeiss Meditec). All visual fields included in the study had fixation losses, false positive and false negative errors less than $20 \%$. Average visual field sensitivity was expressed in MD (mean deviation), as calculated by the perimetry software. A visual field defect was defined as having three or more significant $(P<0.05)$ non-edge contiguous points with at least one at the $P<0.01$ level on the same side of horizontal meridian in the pattern deviation plot and confirmed with at least two consecutive examinations ${ }^{[22,23]}$.

Visual field progression was analyzed with event-based analysis using the Guided Progression Analysis (GPA, Carl Zeiss Meditec) according to EMGT criteria ${ }^{[28]}$. Progression was defined when there were $\geq 3$ points that showed significant changes (greater than the test-retest variabilities) compared with two baseline examinations (separated by $\sim 4$ months in this study) for $\geq 2$ or 3 consecutive tests (i.e. "likely progression" was noted in the GPA printout in the latest follow-up visit).

\section{Statistical Analyses}

Page $12 / 16$ 
Statistical analyses were performed using Stata version 12.0 (Stata Corp LP, College Station, TX). Differences in demographics, visual field, ONH, and RNFL measurements between the progressive and nonprogressive eyes were compared with linear mixed models after adjustment of correlation between fellow eyes. A multivariate cox proportional hazards model was used to investigate if baseline corneal hysteresis and other risk factors including baseline age, CCT, baseline MD, baseline disc area, baseline diastolic IOP, and mean IOP during follow-up were risk factors for progressive RNFL thinning, ONH surface depression and visual field progression. Adjusted HRs from the multivariate cox proportional hazards models were reported in this study. $P<0.05$ was considered statistically significant.

\section{Declarations}

\section{Data availability}

The data used during the current study are available from the corresponding author on reasonable request.

\section{Acknowledgements}

We sincerely acknowledge Prof LEUNG Kai Shun Christopher from Department of Ophthalmology and Visual Sciences, The Chinese University of Hong Kong for supporting this study and revising the manuscript.

\section{Author contributions}

G.X and C.Z designed the study and wrote the manuscript. G.X collected and analyzed the data. C.Z supervised the study and edited the manuscript.

\section{Competing interests}

The authors declare no competing interests

\section{References}

1. Medeiros FA, Susanna R Jr, Singh K. Medical Treatment of Glaucoma, in Who should be treated? Kugler: Amsterdam,The Netherlands: 1-19 (2010).

2. Xu G, Weinreb RN, Leung CK. Optic nerve head deformation in glaucoma: the temporal relationship between optic nerve head surface depression and retinal nerve fiber layer thinning. Ophthalmology. 121(12):2362-70. doi: 10.1016/j.ophtha.2014.06.035 (2014).

3. The Advanced Glaucoma Intervention Study (AGIS): 7. The relationship between control of intraocular pressure and visual field deterioration.The AGIS Investigators. Am J Ophthalmol. 130(4):429-40. doi: 10.1016/ s0002-9394(00)00538-9 (2000). 
4. Kass MA, Heuer DK, Higginbotham EJ, et al. The Ocular Hypertension Treatment Study: a randomized trial determines that topical ocular hypotensive medication delays or prevents the onset of primary open-angle glaucoma. Arch Ophthalmol. 120(6):701-13; discussion 829-30 (2002).

5. Leske MC, Heijl A, Hyman L, et al; EMGT Group. Predictors of long-term progression in the early manifest glaucoma trial. Ophthalmology. 114(11):1965-72. doi: 10.1016/j.ophtha.2007.03.016 (2007).

6. Medeiros FA, Alencar LM, Zangwill LM, Sample PA, Weinreb RN. The Relationship between intraocular pressure and progressive retinal nerve fiber layer loss in glaucoma. Ophthalmology. 116(6):1125-33. e1-3. doi: 10.1016/j.ophtha.2008.12.062 (2009).

7. Miglior S, Zeyen T, Pfeiffer N,et al; European Glaucoma Prevention Study (EGPS) Group. Results of the European Glaucoma Prevention Study. Ophthalmology. 112(3):366-75. doi: 10.1016/j.ophtha. 2004.11.030 (2005).

8. Luce DA. Determining in vivo biomechanical properties of the cornea with an ocular response analyzer. J Cataract Refract Surg. 31(1):156-62. doi: 10.1016/j.jcrs.2004.10.044 (2005).

9. Congdon NG, Broman AT, Bandeen-Roche K, Grover D, Quigley HA. Central corneal thickness and corneal hysteresis associated with glaucoma damage. Am J Ophthalmol. 141(5):868-75. doi: 10.1016/j.ajo.2005.12.007 (2006).

10. De Moraes CV, Hill V, Tello C, Liebmann JM, Ritch R. Lower corneal hysteresis is associated with more rapid glaucomatous visual field progression. J Glaucoma. 21(4):209-13. doi: 10.1097/IJG.0b013e3182071b92 (2012).

11. Medeiros FA, Meira-Freitas D, Lisboa R,et al. Corneal hysteresis as a risk factor for glaucoma progression: a prospective longitudinal study. Ophthalmology. 120(8):1533-40. doi: 10.1016/j.ophtha.2013.01.032 (2013).

12. Jonas JB, Hayreh SS, Tao Y. Central corneal thickness and thickness of the lamina cribrosa and peripapillary sclera in monkeys. Arch Ophthalmol. 127(10):1395-6. doi:

10.1001/archophthalmol.2009.243 (2009).

13. Jonas JB, Stroux A, Oberacher-Velten IM, Kitnarong N, Juenemann A. Central corneal thickness and development of glaucomatous optic disk hemorrhages. Am J Ophthalmol. 140(6):1139-41. doi: 10.1016/j.ajo.2005.06.056 (2005).

14. Prata TS, Lima VC, Guedes LM, et al. Association between corneal biomechanical properties and optic nerve head morphology in newly diagnosed glaucoma patients. Clin Exp Ophthalmol. 40(7):682-8. doi: 10.1111/j.1442-9071.2012.02790.x (2012).

15. Prata TS, Lima VC, de Moraes CG, et al. Factors associated with topographic changes of the optic nerve head induced by acute intraocular pressure reduction in glaucoma patients. Eye (Lond). 25(2):201-7. doi: 10.1038/eye.2010.179 (2011). 
16. Wells, A. P., D. F. Garway-Heath, A. Poostchi, et al. Corneal hysteresis but not corneal thickness correlates with optic nerve surface compliance in glaucoma patients. Invest Ophthalmol Vis Sci. 49(8): 3262-8 (2008).

17. Kotecha A, Elsheikh A, Roberts CR, Zhu H, Garway-Heath DF. Corneal thickness- and age-related biomechanical properties of the cornea measured with the ocular response analyzer. Invest Ophthalmol Vis Sci. 47(12):5337-47. doi: 10.1167/iovs.06-0557 (2006).

18. Sullivan-Mee M, Katiyar S, Pensyl D, Halverson KD, Qualls C. Relative importance of factors affecting corneal hysteresis measurement. Optom Vis Sci. 89(5):E803-11. doi: 10.1097/OPX.0b013e3182504214 (2012).

19. Sigal IA, Flanagan JG, Tertinegg I, Ethier CR. Modeling individual-specific human optic nerve head biomechanics. Part II: influence of material properties. Biomech Model Mechanobiol. 8(2):99-109. doi: 10.1007/ s10237-008-0119-0 (2009).

20. Kotecha A, Elsheikh A, Roberts CR, Zhu H, Garway-Heath DF. Corneal thickness- and age-related biomechanical properties of the cornea measured with the ocular response analyzer. Invest Ophthalmol Vis Sci. 47(12):5337-47. doi: 10.1167/iovs.06-0557 (2006).

21. Kaufmann C, Bachmann LM, Thiel MA. Intraocular pressure measurements using dynamic contour tonometry after laser in situ keratomileusis. Invest Ophthalmol Vis Sci. 44(9):3790-4. doi: 10.1167/iovs.02-0946 (2003).

22. Leung CK, Cheung $\mathrm{CY}$, Weinreb RN,et al. Retinal nerve fiber layer imaging with spectral-domain optical coherence tomography: a variability and diagnostic performance study. Ophthalmology.116(7):1257-63, 1263.e1-2. doi: 10.1016/j.ophtha.2009.04.013 (2009).

23. Yu M, Weinreb RN, Yiu C, et al. Computer simulation of progressive retinal nerve fiber layer loss in glaucoma: performance of event and trend analyses. Invest Ophthalmol Vis Sci. 52(13):9674-83. doi: 10.1167/ iovs.11-8052 (2011).

24. Leung CK, Yu M, Weinreb RN, et al. Retinal nerve fiber layer imaging with spectral-domain optical coherence tomography: patterns of retinal nerve fiber layer progression. Ophthalmology. 119(9):1858-66. doi: 10.1016/j.ophtha.2012.03.044 (2012).

25. Chauhan BC, Blanchard JW, Hamilton DC, LeBlanc RP. Technique for detecting serial topographic changes in the optic disc and peripapillary retina using scanning laser tomography. Invest Ophthalmol Vis Sci. 41(3):775-82 (2000).

26. Chauhan $\mathrm{BC}$, Hutchison DM, Artes $\mathrm{PH}$, et al. Optic disc progression in glaucoma: comparison of confocal scanning laser tomography to optic disc photographs in a prospective study. Invest Ophthalmol Vis Sci. 50(4):1682-91. doi: 10.1167/iovs.08-2457 (2009).

27. Chauhan BC, Nicolela MT, Artes PH. Incidence and rates of visual field progression after longitudinally measured optic disc change in glaucoma. Ophthalmology. 116(11):2110-8. doi: 10.1016/j.ophtha.2009.04.031(2009). 
28. Heijl A, Leske MC, Bengtsson B, Bengtsson B, Hussein M; Early Manifest Glaucoma Trial Group. Measuring visual field progression in the Early Manifest Glaucoma Trial. Acta Ophthalmol Scand. 81(3):286-93. doi: 10.1034/j.1600-0420.2003.00070.x (2003).

\section{Figures}

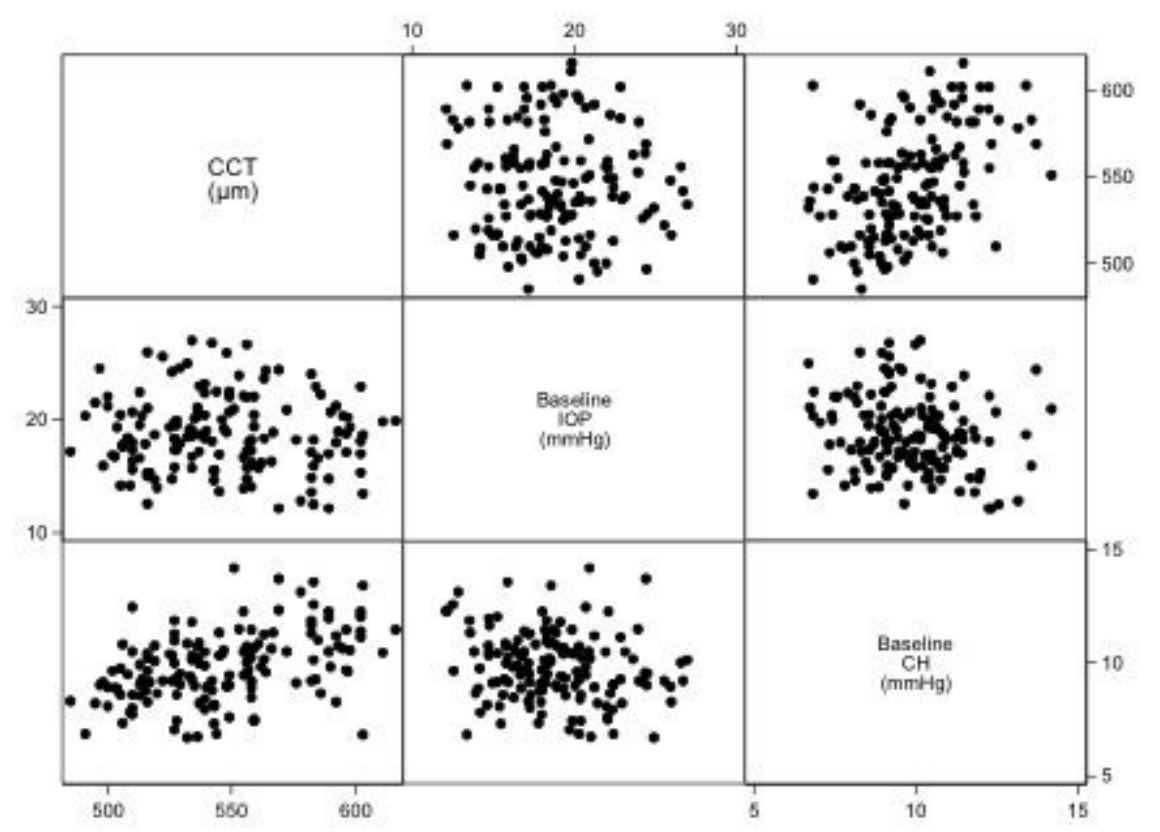

Figure 1

Scatter matrix illustrating the relationship among baseline corneal hysteresis $(\mathrm{CH})$, central corneal thickness (CCT), and baseline dynamic contour tonometry (DCT) measurements. CCT= central corneal thickness; IOP=intraocular pressure. 